\title{
Quantum and high-sensitive laser technologies for polarization-optical diagnostics
}

\author{
Yakov Fofanov* \\ Institute for Analytical Instrumentation RAS, 190103 St. Petersburg, Russia
}

\begin{abstract}
The basics for highly sensitive laser and quantum technologies of polarization-optical measurements and diagnostics of ordered structures, nonlinear interfaces and nanosystems are considered. Quantum diagnostics for a nonlinear resonant beam splitter is performed. It is shown that this beam splitter operates as an optical buffer that reduces the quantum (photon) fluctuations of the reflected light.
\end{abstract}

The prospects for the development and application of highly sensitive laser and quantum measurement and diagnostic technologies are limited in many cases by the lack of sufficiently complete conceptions about their potential possibilities. This article discusses the probing of ordered structures, nonlinear interfaces and nanosystems by laser radiation with deep polarization modulation [1-3].

One of the studied objects were the systems (ensembles) of magnetic nanoparticles in a liquid matrix (magnetic nanofluids, [4,5]). In fig. 1, the dots show the characteristic dependence of the polarization response $\varphi$ on the dimensionless parameter $p$ for a concentration of magnetite nanoparticles in kerosene of $1 \%$ [5]. These experimental data were approximated by a theoretical dependence following from the model of orientational ordering of magnetic particles in an external magnetic field [6]. The solid curve in Fig. 1 shows the results of the approximation, and the inset gives the deviations $\delta$ of the approximation data from the experiment. According to the theoretical model [6], the found values of the dimensionless parameter $\mathrm{p}$ give the opportunity to determine some (unknown) physical parameters by the observed polarization responses and the values of other (known) parameters. Statistical analysis also showed the possibility of revealing subtle features of magnetic nanofluids from small deviations of variable dimensionless parameters.

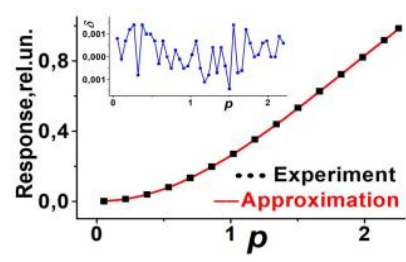

Fig. 1. Magnetic nanofluid

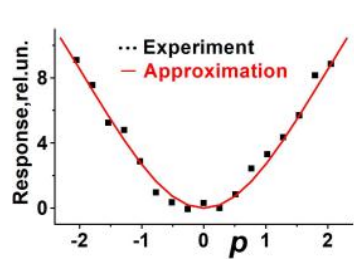

Fig. 2. Iron borate

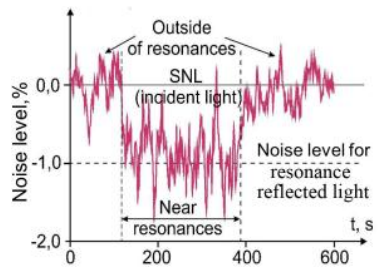

Fig. 3. Quantum optical buffer

\footnotetext{
* Corresponding author: yakinvest@yandex.ru
} 
Another very interesting model object for analysis is iron borate $\left(\mathrm{FeBO}_{3}\right.$, [7]). Studies of $\mathrm{FeBO}_{3}$ have also shown the perspectives of developing highly sensitive laser polarizationoptical diagnostics. The dots in fig. 2 shows the average response of a sample of $\mathrm{FeBO}_{3}$. The solid curve shows the result of the approximation performed similarly to the approximation of the magnetic fluid responses described above. In this case, the dimensionless parameter $p$ should be understood as some effective value characterizing the studied ordered system. Considering this, it is clearly seen that the dependences in Fig. 1 and Fig. 2 are very similar. This indicates a certain similarity of the magnetic behavior of these two systems with visibly different ordering mechanisms [5,7].

Fig. 3 illustrates the possibility of performing quantum polarization-optical diagnostics of a completely different type of system - a nonlinear beam splitter [8,9]. The use of a nonlinear beam splitter for preparing quantum (squeezed) states of light was first proposed by A.V. Belinsky [10].

The nonlinear resonant reflection was experimentally realized from the "glass-rubidium vapor" interface at incidence angles close to the angles of total internal reflection $[8,9]$. The selective (resonant) reflection at large angles of incidence has significant advantages, because it gives rather strong reflective resonances, which are saturated at relatively weak light fields $[11,12]$.

Fig. 3 shows a decrease of quantum (photon) fluctuations in reflected light. In other words, this beam splitter operates like a quantum optical buffer. The effective thickness of the active region is about $0.1 \mu \mathrm{m}$, i.e., the realized configuration is characteristic for nanophotonics. Adequate theoretical analysis confirms the results of the performed polarization-optical nanodiagnostics of the considered nonlinear beam splitter $[13,14]$.

Thus, the basics are developed and the possibility of using quantum and highly sensitive laser technologies of polarization-optical diagnostics for research and quantitative characterization of objects and systems of completely different nature has been shown [5,7$9,15]$.

The work was performed in accordance with State Assignment No. 075-00780-19-02 (theme No. 00742019-0007) of the Ministry of Education and Science of the Russian Federation.

\section{References}

1. J. Badoz, B.M. Billardon, J.C. Canit, M.F.J. Russel, J. Optics (Paris). 8(6), 373 (1977)

2. Ya. A. Fofanov, Proceedings SPIE, 1811, 413 (1992)

3. I. M. Sokolov, Ya. A. Fofanov, J. Opt. Soc. Am., A 12, 1579 (1995)

4. C. Scherer, A.M. Figueiredo Neto, Brazilian Journ. of Physics. 35(3A), 718 (2005)

5. Ya.A. Fofanov, I.V. Pleshakov, A.V. Prokof'ev, E.E. Bibik, Technical Physics Letters, 42, 1054 (2016),

6. Scholten P.C. IEEE Trans. Magn., 16(2), 221 (1980)

7. Ya.A. Fofanov, I.V. Pleshakov, Yu.I. Kuz'min, J. Opt. Technol., 80, 64 (2013)

8. Fofanov Ya.A. Opt. and Spectr., 94, P. 802 (2003)

9. Ya. A. Fofanov, In “Advances in Optoelectronics Research”, P. 75-114, Ed. Marcus R. Oswald (Nova Science Publishers, Inc., USA, 2014)

10. A.V. Belinskii, JETP Lett., 51, 387 (1990)

11. Ya.A. Fofanov, Quantum Electronics 39(6), 585 (2009)

12. V.A. Sautenkov, H. Li, M.A. Gubin, Yu.V. Rostovtsev, M.O. Scully, Laser Physics, 21 (1), 153 (2011)

13. A.V. Belinsky, D.V. Volkov, A.V. Dmitriev, M.Kh. Shulman, JETP, 117, 771 (2013)

14. A.V. Belinsky, M.Kh. Shulman, Phys-USP, 57, 1022 (2014)

15. Ya.A. Fofanov, J. Opt. Technol., 87 (2020). (to be published) 